\title{
Gastric Perforation after Cesarean Delivery: An Unintended Consequence of the Opioid Epidemic
}

\author{
Tesia A. McKenzie ${ }^{1}$, Eugene Zurkovsky², Jonathan D. Baum ${ }^{1}$ \\ ${ }^{1}$ Department of Obstetrics and Gynecology, Departments Affiliated with Jersey Shore University Medical Center, Hackensack \\ Meridian Health System, Neptune, USA \\ ${ }^{2}$ Department of General Surgery, Departments Affiliated with Jersey Shore University Medical Center, Hackensack Meridian \\ Health System, Neptune, USA \\ Email: jonathan.baum@hmhn.org
}

How to cite this paper: McKenzie, T.A., Zurkovsky, E. and Baum, J.D. (2021) Gastric Perforation after Cesarean Delivery: An Unintended Consequence of the Opioid Epidemic. Open Journal of Obstetrics and Gynecology, 11, 563-568.

https://doi.org/10.4236/ojog.2021.115052

Received: March 24, 2021

Accepted: May 16, 2021

Published: May 19, 2021

Copyright (c) 2021 by author(s) and Scientific Research Publishing Inc. This work is licensed under the Creative Commons Attribution International License (CC BY 4.0).

http://creativecommons.org/licenses/by/4.0/

\begin{abstract}
30 year old female now para 1 presented to the Emergency Department with nausea, vomiting, and abdominal pain 6 days after an uncomplicated primary cesarean delivery. She did not respond to conservative management and underwent exploratory laparotomy for worsening pain, pneumoperitoneum and intraabdominal fluid collections. Gastric perforations required repair via gastrojejunostomy. Postoperative course was unremarkable. The anti-opioid campaign has altered the approach to postoperative pain management in both positive and negative ways. It has sparked new interest in alternative approaches to postoperative pain management, which include an increased role for non-steroidal anti-inflammatory drugs (NSAIDs). We present a case of a woman who had a significant complication due to the reliance of nonopioid pain medications after cesarean delivery.
\end{abstract}

\section{Keywords}

Opioid, Opioid Epidemic, Gastric Perforation, Gastrojejunostomy, Cesarean Delivery

\section{Introduction}

The widespread campaign to increase awareness of the opioid crisis has led to a growing antipathy toward the use of opioids for postoperative pain. Public health experts have issued constant reminders about the death toll due to opiates and how medical professionals should be addressing this crisis [1] [2]. The New Jersey Board of Medical Examiners has limited initial opioid prescriptions to a 
maximum of 5 days given the association between number of prescribed and addiction. Patients and clinicians are now fearful of the appropriate opioid prescribing and use.

Despite ample evidence that progression to prolonged opioid use following obstetric and gynecologic surgery is low (ranging from $0.03 \%-0.2 \%$ ), both reduced and non-opioid based methods of postoperative pain management are being promoted [1] [3] [4]. These new approaches rely heavily on NSAIDs [5]. However, NSAIDs are not without risk and have been associated with dyspepsia, gastrointestinal bleeding, ulceration and perforation [6]. In addition, reluctance to prescribe opioids has been associated with the under treatment of postoperative pain [7].

The opioid crisis has created a unique dilemma in regard to use of opioids to relieve pain following surgery; they are feared, yet remain essential [8]. We present a case of a young woman who was harmed due to a reliance on non-opioid pain management following cesarean delivery. The patient consented to presentation/publication of her case prior to discharge from the hospital.

\section{Case}

A healthy 30 year old female now para 1 presented to the Emergency Department with nausea, vomiting, and abdominal pain 6 days after an uncomplicated primary cesarean delivery. Her medical history was significant for endometriosis and diagnostic laparoscopy. She had no known drug allergies and was taking ibuprofen and acetaminophen for postoperative pain. She did not smoke, drink alcohol or use illicit drugs. Her white blood count was $12,000 / \mu \mathrm{L}$ and she was afebrile. CT scan of the abdomen and pelvis showed pneumoperitoneum and an enlarged uterus consistent with recent obstetric surgery (Figure 1). The differential diagnosis included postoperative ileus, endometritis and bowel injury. She was managed conservatively with IV fluids, bowel rest and decompression via nasogastric tube. She initially improved, however her abdominal pain and

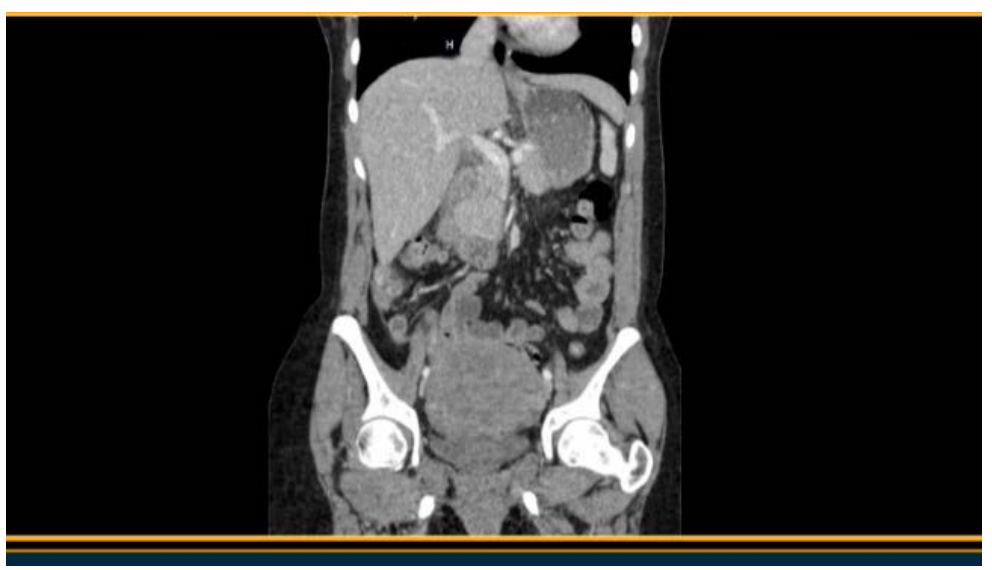

Figure 1. CT abdomen and pelvis showing small pneumoperitoneum thought to be secondary to patient's most recent surgery. There is also a small amount of ascites, enlarged heterogeneous postpartum uterus. No evidence of bowel obstruction. 
leukocytosis worsened $(18,000 / \mu \mathrm{L})$ over the following 72 hours. She did not respond to empiric broad spectrum antibiotics. Exploratory surgery was recommended, however she declined. She refused opioid pain medications out of fear of becoming addicted.

On hospital day 7, repeat CT scan now showed worsening pneumoperitoneum with a significant amount of air surrounding the liver and anterior to the stomach (Figure 2(a), Figure 2(b)). Given her worsening abdominal pain, leukocytosis and imaging and lack of response to conservative management, she agreed to surgery.

Exploratory laparotomy showed a $3 \mathrm{~cm}$ gastric perforation of both the anterior and posterior walls with perihepatic peritonitis (Figure 3). She underwent repair via gastro jejunostomy (Billroth 2 procedure) [9]. Her postoperative course was unremarkable, her pain resolved and she was discharged to home in stable condition 6 days after surgery. Her post hospital course was uncomplicated and she was doing well at the time of this writing.

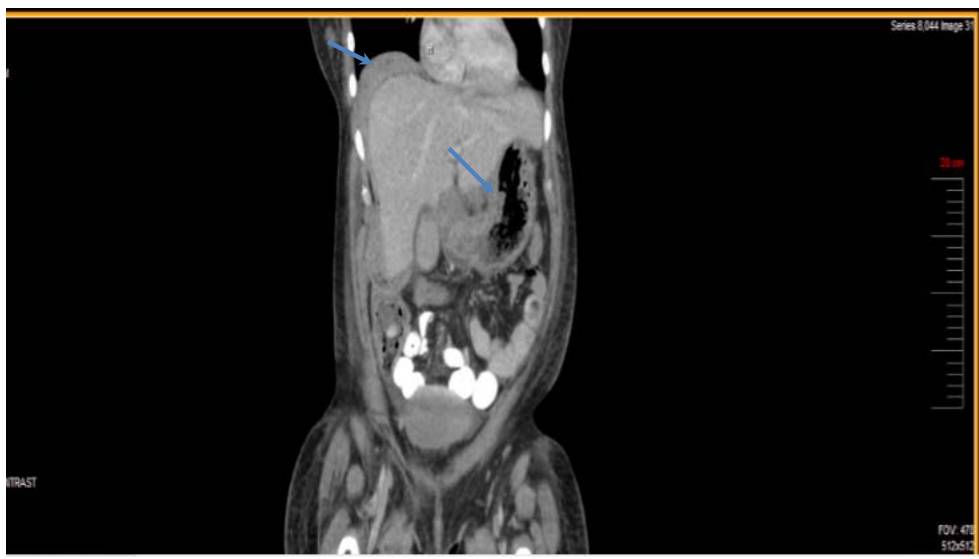

(a)

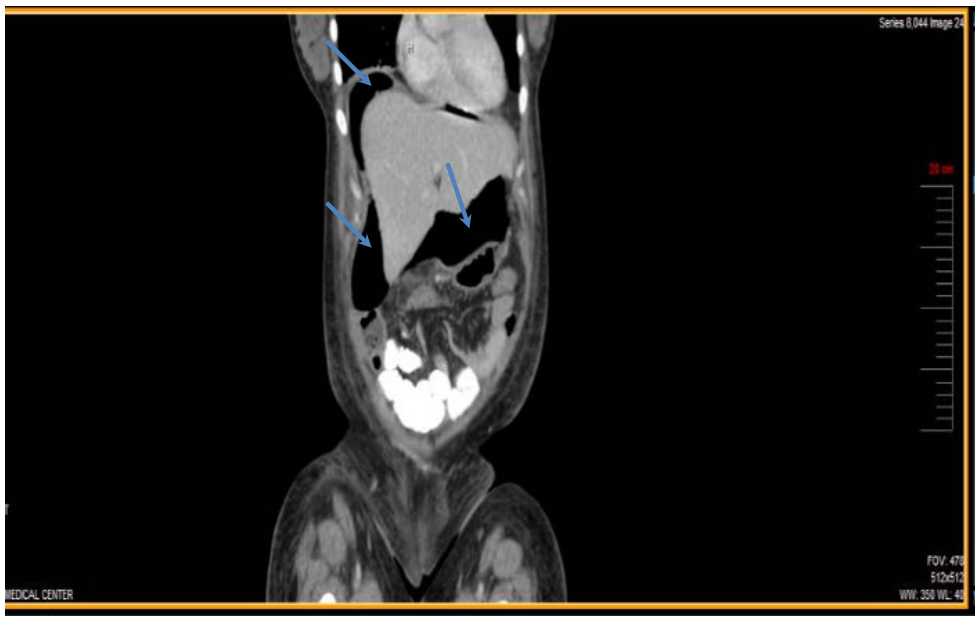

(b)

Figure 2. Parts (a) and (b) illustrate the patient's repeat CT Abdomen and Pelvis showing the worsening pneumoperitoneum with epigastric and perihepatic air and fluid collections. 


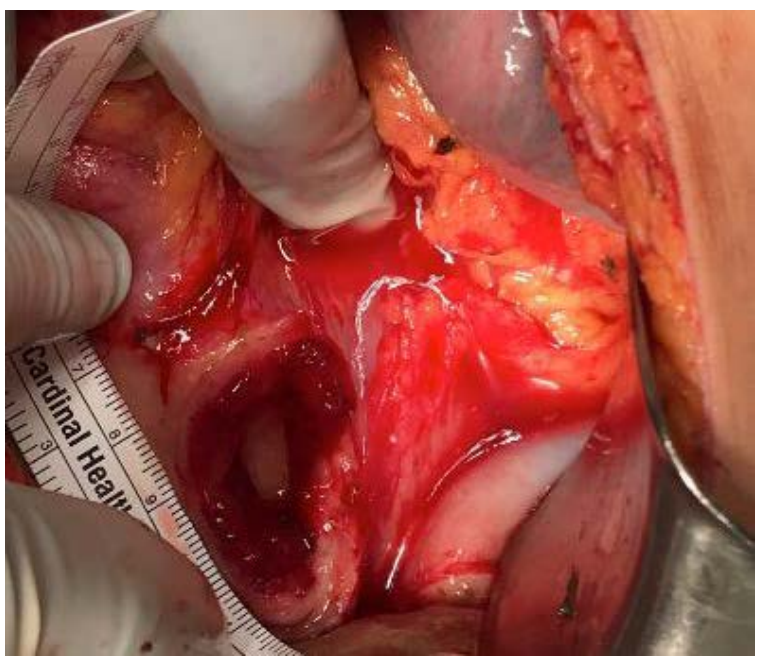

Figure 3. Image of the patient's stomach showing the $3 \mathrm{~cm}$ perforation through the anterior and posterior walls.

\section{Discussion}

The opioid crisis has led us to re-examine the management of postoperative pain. This increased awareness has led to the promotion of a multimodal approach to analgesia that relies heavily on NSAIDs [5]. However, these medications are not without risk and have been associated with dyspepsia, gastrointestinal bleeding, ulceration and perforation [6]. Our patient used NSAIDs extensively out of fear of developing an opioid addiction despite having no risk factors for misuse or abuse. She developed a gastric perforation, which required a major corrective surgery (gastrectomy), which has lifelong consequences. Following gastrectomy, patients are at increased risk for short and long term complications such as leakage, stricture and ulcer. Motility issues such as dumping syndrome (carbohydrate intolerance) may require lifelong dietary changes [9].

The anti-opioid message must be crafted to maximize societal awareness and minimize misunderstanding and bias. There is strong evidence to suggest that women can use opioids appropriately following OB/GYN surgery without increasing their risk of addiction. According to the American College of Obstetricians and Gynecologists (ACOG) $0.3 \%$ ( 1 in 300) of patients who take opioids after cesarean delivery will become persistent users [1]. Young et al. (2020) reported the risk of prolonged opioid use following hysterectomy was less than $0.07 \%$ ( 1 in 1538) [3]. Additionally, Sun et al. (2016) reported the incidence of chronic opioid use at 1 year following cesarean delivery was less than $0.2 \%$ ( 1 in 500) [4].

It is estimated that $1 \%-2 \%$ of continuous NSAID users experience a significant upper GI complication ranging from bleeding to obstruction and perforation [6]. We agree that NSAIDs should have a significant role in postoperative pain management however, not at the exclusion of opioids. This patient may have overestimated her risk of opioid addiction while underestimating the risks of continuous NSAID use. This highlights the importance of a balanced ap- 
proach to postoperative pain management.

The anti-opioid discussion has focused mainly on the risks of opioids with little to no attention being paid to the potential downside of the alternatives. Given the growing bias against opioids, it is possible that complications of the alternatives are being under-reported. With the exception of NSAIDs, we could not find commentary or editorials questioning the rapid adoption of non-opioid based methods of postoperative pain management. Overstating the risk of addiction while understating the risks of the alternative may have led to the complication we present. This case highlights an unintended consequence of the opioid epidemic.

\section{Conclusion}

Opioids can remain an important and safe component of postoperative pain management with very low rates of addiction. Given that more than $99 \%$ of women can use opioids appropriately in the postoperative setting, limiting opioid use for every patient may not be justified.

\section{Conflicts of Interest}

The authors declare no conflicts of interest regarding the publication of this paper.

\section{References}

[1] NIDA (2020) Emphasis Is Needed on Both Prevention and Treatment. National Institute on Drug Abuse.

https://www.drugabuse.gov/publications/research-reports/prescription-opioids-her oin/emphasis-needed-both-prevention-treatment

[2] Volkow, N.D. and Collins, F.S. (2017) The Role of Science in Addressing the Opioid Crisis. The New England Journal of Medicine, 377, 391-394. https://doi.org/10.1056/NEJMsr1706626

[3] Young, J.C. et al. (2020) Persistent Opioid Use after Hysterectomy in the United States, 2005-2015. Obstet \& Gynecol, 135, 123-132. https://doi.org/10.1097/AOG.0000000000003612

[4] Sun, E.C., Darnall, B.D., Baker, L.C. and Mackey, S. (2016) Incidence of and Risk Factors for Chronic Opioid Use among Opioid-Naive Patients in the Postoperative Period. JAMA Internal Medicine, 176, 1286-1293. https://doi.org/10.1001/jamainternmed.2016.3298

[5] American College of Obstetricians and Gynecologists (2018) ACOG Committee Opinion No. 742: Postpartum pain management. ObstetGynecol 2018 May 17; [e-pub].

[6] Goldstein, J.L. and Cryer, B. (2015) Gastrointestinal Injury Associated with NSAID Use: A Case Study and Review of Risk Factors and Preventative Strategies. Drug, Healthcare and Patient Safety, 7, 31-41. https://doi.org/10.2147/DHPS.S71976

[7] Bowen, K.R. and Baum, J.D. (2017) Life after Addiction-Post-Operative Pain Management in an Obstetrical Patient on Long-Term Buprenorphine Therapy. Open Journal of Obstetrics and Gynecology, 7, 951-956.

https://doi.org/10.4236/ojog.2017.79096 
[8] Rosenblum, A., Marsch, L.A., Joseph, H. and Portenoy, R.K. (2008) Opioids and the Treatment of Chronic Pain: Controversies, Current Status, and Future Directions. Experimental and Clinical Psychopharmacology, 16, 405-416. https://doi.org/10.1037/a0013628

[9] Hebbard, P., Soybel, D.I. and Chen, W. (2019) Partial Gastrectomy and Gastrointestinal Reconstruction.

https://www.uptodate.com/contents/total-gastrectomy-and-gastrointestinal-reconst $\underline{\text { ruction? } \text { topicRef }=15161 \text { \&source }=\text { see link }}$ 